Meta

Journal des traducteurs

Translators' Journal

\title{
Regards sur le vocabulaire du rail
}

\section{Gérard de Jaham}

Volume 14, numéro 1, mars 1969

URI : https://id.erudit.org/iderudit/003238ar

DOI : https://doi.org/10.7202/003238ar

Aller au sommaire du numéro

Éditeur(s)

Les Presses de l'Université de Montréal

ISSN

0026-0452 (imprimé)

1492-1421 (numérique)

Découvrir la revue

Citer cet article

de Jaham, G. (1969). Regards sur le vocabulaire du rail. Meta, 14(1), 34-38.

https://doi.org/10.7202/003238ar d'utilisation que vous pouvez consulter en ligne.

https://apropos.erudit.org/fr/usagers/politique-dutilisation/ 
compétence des problèmes de leur spécialité. Souvent, trop souvent, ils s'expriment dans une langue effroyable, déformée par les anglicismes. La raison, tout le monde la connaît, puisque, à une époque très récente, il fallait aller parfaire ses études aux États-Unis et que, même encore aujourd'hui, on fait des études supérieures en utilisant des manuels de langue anglaise. C'est donc aller très souvent à une catastrophe que de confier la traduction d'un manuel scolaire à l'un des plus éminents spécialistes de sa discipline.

Pour remédier à cette situation, on a proposé d'adjoindre à ces spécialistes un linguiste qui serait chargé de remettre en bon français le premier jet du traducteur. Mais une telle dualité ne va pas sans heurts!

Les spécialistes tiennent en effet dur comme fer à leur terminologie, même si elle constitue un jargon, et cela pour une raison bien simple, c'est que l'esprit préfère utiliser, pour raisonner, un outil imparfait qu'il connaît bien à un outil parfait mais inconnu.

Le linguiste, lui, ne s'intéresse qu'à la langue, au point même de se désintéresser des nécessités pédagogiques. Il pourchassera par exemple l'anglicisme au point de reconstruire des expressions usuelles, claires et foncièrement correctes parce que, par hasard, elles sont calquées sur la phrase anglaise correspondante. Entre le spécialiste furieux qu'on «abîme » son texte et le linguiste prêt à défendre la langue française jusqu'à la mort, l'éditeur un beau jour se voit forcé d'être arbitre car il y a des impératifs de date de parution qui exigent de mettre fín à ce genre de discussion.

Un mot, pour terminer, du coût des traductions. Il est trop élevé. Les traducteurs professionnels ont été amenés à établir un tarif au mot qui se comprend parfaitement puisque $90 \%$ des traductions « anglais-français », «français-anglais » qui se font dans le Québec sont des textes commerciaux: annonces, communiqués, circulaires, etc. De là à vouloir appliquer ce tarif au mot lorsqu'il s'agit d'un livre, il y a un abîme.

Aussi longtemps que ce problème ne sera pas résolu, on continuera pour traduire des livres à se passer des services de traducteurs professionnels, en faveur d'amateurs qui, s'ils sont consciencieux, passeront sur les pages d'un livre quatre ou cinq fois plus de temps qu'il n'en faudrait à un traducteur professionnel.

$\mathrm{Si}$ j'ai énuméré les difficultés, c'est parce qu'elles me paraissent d'une extrême gravité et qu'il semble que bien souvent on les méconnaisse. Elles sont difficiles à surmonter mais on y parvient, puisque nous avons vu récemment un écrivain de la qualité de Jean Simard prêter son talent à Hugh MacLennan et que d'excellentes traductions de manuels scolaires ont été réalisées dernièrement.

Il y a cependant du chemin à faire pour que chez nous le mot traduction n'ait pas un sens péjoratif et qu'on puisse l'employer sans qu'inconsciemment votre interlocuteur le fasse précéder de l'adjectif «mauvais».

Pierre Tisseyre 


\section{PROBLÈMES ET SOLUTIONS}

\section{REGARDS SUR LE VOCABULAIRE DU RAIL}

L'histoire des chemins de fer commence il y a environ un siècle et demi. Bien que l'on ait voulu reconnaître certains types de chars romains comme ancêtres de nos voitures modernes et que l'image de rails la plus ancienne remonte à 1550 , c'est au début du $\mathrm{XIX}^{\mathrm{e}}$ siècle que des véhicules montés sur roues à boudins (flanged wheels) commencèrent à circuler sur des rails.

Et de l'antique "chemin à lisse » au ruban d'acier moderne, que de progrès accomplis et quel enrichissement pour notre langue technique !

Le développement du matériel ferroviaire, en particulier du matériel de traction, s'accomplissant plus rapidement en Angleterre qu'en France, il n'est pas étonnant que notre langue ait dû adopter tout d'abord certains termes anglais: tramways, wagons, etc.; la fameuse locomotive «Crampton» devint même synonyme de train en France, vers les années 1850. Par contre, si le mot rail nous est revenu d'Angleterre vers 1825, son origine française est indiscutable puisqu'il dérive des vieux mots riceule et rieulet désignant, en patois du Hainaut, une règle de maçon qui s'appelait également rhil en vieux français, Regel ou Riegel en bas allemand.

Bien vite cependant, les ingénieurs français se distinguent dans certains domaines, en particulier celui de la signalisation et plus tard dans celui de la pose et de l'entretien de la voie. Aussi notre langue dispose-t-elle aujourd'hui d'un vocabulaire riche, précis et souvent imagé, pour décrire les éléments nombreux et complexes de cette branche de l'industrie des transports.

Présenter ce vocabulaire, analyser les différentes expressions, n'est pas chose facile, tant la matière est abondante: le seul lexique des termes ferroviaires publié par l'U.I.C. en six langues compte plus de 11000 références et laisse volontairement de côté l'aspect administratif et syndical, particulier à chaque pays.

Suivant ce sage exemple, nous nous bornerons à évoquer ici l'aspect technique de la langue du cheminot. 
Nos trains circulent en vertu d'horaires précis, sur des voies où l'automatisme joue un rôle de plus en plus grand, au service de la sécurité. De quoi se composent-ils donc?

Il y a lieu de distinguer trois catégories de matériel roulant: le matériel de traction (motive power), le matériel voyageurs (passenger equipment) et le matériel marchandises (freight equipment). Les locomotives diesels, dieselsélectriques et électriques ont pris la relève de nos bons «teuf-teuf » à vapeur. Plus puissantes, plus rapides, elles sont aussi d'une utilisation plus souple. Certaines circulent sur les grandes lignes, ce sont en général les diesels, d'autres sur les lignes intervilles ou de banlieue; celles-ci sont purement électriques et s'approvisionnent en courant force à une caténaire (catenary ou overhead contact line) grâce à leur pantographe (pantograph ou current collector). Affectées à une gare de triage, elles prennent le nom de locomotives de mancuvre (yard. engine), et lorsqu'elles se déplacent sans rien pousser ni tirer on dit qu'elles circulent haut-le-pied (deadheading).

À ces engins modernes viennent s'atteler les véhicules destinés aux voyageurs et qui sont les voitures (passenger cars). Ce mot, adopté par l'U.I.C. par opposition à wagons (freight cars), permet de distinguer clairement les services voyageurs des services marchandises. Mais alors, le wagon-lit? Précisons que dans tous les cas, il convient d'employer voiture (voiture-lit, voiture-restaurant, voiture-salon, etc.). L'appellation wagon-lit disparaît et des démarches auraient même été entreprises auprès de la fameuse Société des wagons-lits Cook pour faire adopter le mot voiture dans sa raison sociale. Nous ne connaissons pas le résultat de ces démarches; il semblerait que pour des raisons publicitaires évidentes, la société soit réticente à accepter le changement proposé. Toutefois, sur le matériel lui appartenant, sont inscrits les mots: voitures-lits - sleeping-car - schlaffwagen - carozzaletti ou coche-camas. Les voitures sont de différents types, correspondant généralement aux anciennes classes. Une difficulté s'est présentée lorsqu'il fallut rendre en français le mot coach. L'appellation voiturecoach est-elle correcte, alors que coach veut dire voiture? Nous butons certes sur une forme pléonastique. En France, on avait contourné la difficulté en désignant ce matériel sous le vocable de «voitures américaines». Mais, au Canada, nous n'avons que des voitures américaines. Ce qu'il faut, c'est différencier pour des raisons commerciales évidentes, les véhicules voyageurs d'après l'aménagement qu'ils comportent, sans parler de «classes». Je serais en faveur de l'adoption de «coach» en français, d'autant plus que certains fabricants d'automobiles n'hésitent pas à présenter leurs produits au salon d'automne à Paris, sous des désignations telles que "break », " roadster », etc. Dans le domaine de la vente et de la publicité, chacun sait quelle bonne fortune est faite au mot étranger. Et puisqu'il faut faire une concession, faisons-la au snobisme plutôt qu'à la médiocrité.

Cette brève revue du matériel voyageurs serait incomplète si nous ne disions quelques mo's du train de l'avenir, le Turbotrain. Rappelons la fiche terminologique publiée dans cette même revue sur ce sujet ${ }^{1}$. L'une des particularités du Turbotrain réside dans le fait que les motrices ont été aménagées de façon à

1. Cf. MeTA, XIII, 4: 194-195.

Vol. $14-\mathrm{N}^{\circ} 1$

Mars 1969 
recevoir des voyageurs. Attelées aux deux extrémités de la rame, elles offrent un compartiment surélevé et généreusement vitré, ce qui leur vaut le nom de motrice panoramique ( $p o w e r$ dome car). Train de composition fixe, le «Turbo » se distingue par l'originalité de sa suspension pendulaire, par la réduction du nombre des essieux (chaque essieu reçoit les extrémités de deux caisses voisines). Enfin la rame est dite réversible (bi-directional).

Pour servir ses clients du secteur marchandises, une compagnie de chemin de fer se doit, de nos jours, de leur offrir un matériel diversifié et au besoin spécialisé. Le parc des Chemins de fer nationaux, par exemple, se compose de quelque 110000 wagons, et il est intéressant de signaler la publication par cette société d'un catalogue de matériel marchandises en anglais et en français. La traduction de cette brochure s'appuie sur une documentation intéressante dont nous citons en note les références bibliographiques ${ }^{2}$. On y trouve une documentation simple et de consultation facile sur les différents types de wagons utilisés, depuis le tombereau (gondola car) jusqu'au wagon porte-auto (autotransporter). Relevons au passage, dans la série des wagons à température variable, la distinction que fait le français entre les adjectifs isotherme, frigorifique et réfrigérant que l'anglais rend par insulated car, mechanical refrigerator et non-mechanical refrigerator. Il y a incontestablement gain et économie en français.

Je ne citerai que quelques modèles de wagons couramment utilisés : wagons plats (flat cars), wagons à parois de bout en $\mathbf{U}$ (flat cars with wraparound bulkheads), wagons à ranchers (flat cars with stakes), utilisés notamment pour le transport du bois en rondins ou en planches, wagons-trémies (hopper cars), wagons porte-autos à deux ou trois ponts (bi-level ou tri-level auto-transporters), wagons couverts à porte encastrée (plug-door cars), sans compter les wagons spécialisés adaptés au transport de produits industriels bien précis : porte-tourets (reel-car), wagon-poche (ladle-car), etc.

Dans le secteur des messageries, on trouve principalement les remorques rail-route (piggyback trailers) qui constituent le gros du matériel.

Voilà un bref aperçu du matériel roulant utilisé pour l'exp'oitation des trois secteurs traditionnels, le secteur voyageurs, le secteur marchandises et le secteur messageries. Ces trois termes sont adoptés universellement, et la distinction entre «Petite vitesse », "Grande vitcsse » ou « Régime accéléré » et « Régime ordinaire » tend à disparaître devant l'intégration des divers services de transport de marchandises de détail.

Nos trains sont maintenant prêts à se lancer sur les mailles d'un réseau où sont aménagées les différentes installations fixes (facilities): gares de voyageurs ou de marchandises, gares de triage, haltes, etc. De quoi se composent ces voies et comment sont-elles faites? La voie exige, pour être posée, un grand nombre de travaux et d'opérations effectués par des spécialistes: tracés, relevés géodésiques, percement de tunnels, construction de viaducs et autres ouvrages d'art, préalables à la constitution de l'assise (road bed). Une fois cette assise

2. La traduction du catalogue de matériel marchandises s'appuie sur la publication du Syndicat des constructeurs français de matériel roulant de chemin de fer, intitulée Matériel roulant de chemin de fer, sur divers articles de la Vie du rail et sur l'ouvrage américain Railway Car Cyclopedia. 
préparée, on procède au ballastage (ballasting) puis à la pose des traverses (tie laying) qui recevront à leur tour les rails. Divers types de rails ont été essayés et utilisés. Les plus répandus de nos jours sont des barres d'acier au profil classique en I comportant un champignon (head of rail), une âme (web of the rail) et un patin (flange ou base of rail). De longueur variable, espacés par un joint de dilatation, ils sont progressivement remplacés par les longs rails soudés, mis au point et adoptés en France depuis quelques années et dont la pose exige un matériel de voie très spécialisé et de grand rendement. Sans vouloir procéder à une énumération fastidieuse de ce matériel, il faut mentionner les portiques automoteurs munis de poutre traveleuse qui peuvent «pondre» la voie toute montée sur le ballast. Les rails, une fois posés, sont éclissés et assujettis aux traverses par des crampons ou des tirefonds. Parfois, il peut être nécessaire d'ancrer (anchor) le rail grâce à un dispositif anticheminant (anticreep device) qui l'empêche de se déplacer sous l'effort constant des véhicules. Une voie unique est dite banalisée (two-way track) lorsqu'elle est ouverte à la circulation dans les deux sens.

Rappelons l'existence des passages à niveau, désignés longtemps au Canada par l'expression «traverse de chemin de fer», bonne vieille traduction du railway crossing. Cette expression fautive a aussi l'inconvénient d'être un faux ami, la traverse de chemin de fer (tie) étant cette pièce de bois imprégné sur le travers de laquelle repose le rail. Aujourd'hui, passage à niveau est reconnu comme étant le terme propre et son usage devrait se répandre.

Aux embranchements, à l'approche des gares et dans les triages, la voie est raccordée aux différents aiguillages qui permettront de diriger les trains, de les remiser ou de les " gerber » (terme employé dans les gares de triage pour désigner l'opération de débranchement, les wagons partant sur des voies en faisceaux qui offrent ensuite l'aspect d'une gerbe). Cette suite de vocables nous montre bien que la langue technique française peut être imagée tout en étant précise.

De nos jours, le mouvement des trains est de plus en plus automatique. Ce n'est pas un hasard, donc, si le deuxième symposium de cybernétique ferroviaire s'est déroulé au mois d'octobre 1967 à Montréal, sous la présidence du secrétaire général de l'U.I.C., et à l'invitation des Chemins de fer nationaux du Canada.

Les problèmes de traduction que posaient les communications furent nombreux. Précieusement aidé par les travaux effectués dans d'autres administrations et grâce à la collaboration d'un ingénieur du Service de recherche et de développement, le bureau de traduction du $\mathrm{CN}$ assura la traduction de cinq communications.

Les progrès techniques et l'application des ordinateurs et de la cybernétique aux chemins de fer obligeait les traducteurs à pénétrer un domaine assez ardu, d'autant plus, il faut le reconnaître, que notre langue s'est contentée d'adopter ou de traduire servilement les termes anglais, l'informatique étant de développement récent. Les retards imposés à l'Europe par la guerre ont obligé les techniciens à parer au plus pressé et l'on a adopté «environnement 》 pour environment, «procédure» pour procedure, «circuits logiques 》 pour logical circuits (alors qu'il s'agit de l'un des éléments analytiques de l'ordinateur), etc. 
Souhaitons seulement que le temps permette à ce vocabulaire barbare de décanter et, à ceux qui en ont la charge, de fixer les termes français précis qui doivent normalement correspondre aux termes anglais. L'effort est amorcé tant au Canada qu'en France. Le génie de la langue se retrouve, aère le vocabulaire et le complète. Data processing system, par exemple, se rend par informatique. S'insérant dans la famille des mots qui désignent les sciences ou les techniques, le terme français a l'avantage d'être plus court que l'anglais et recouvre, à mon sens, une aire sémantique plus vaste puisqu'il évoque non seulement les procédés existants mais toute la science de l'information mécanographique. Signalons également les études faites sur les mots hardware et software dont les résultats ont paru dans MeTA ${ }^{3}$.

La langue ferroviaire est sans doute l'une des plus riches et des plus imagées des langues techniques. Vivante et en perpétuelle évolution, elle se doit de refléter l'exactitude et la souplesse qui font la renommée de nos chemins de fer.

GÉRARD DE JAHAM

3. Cf. META, XII, 4: 126; XIII, 2: 73; XIII, 4: 198. 